Pacific Journal of Mathematics

NONCOMPACTNESS PRINCIPLES IN NONLINEAR OPERATOR 


\title{
NONCOMPACTNESS PRINCIPLES \\ IN NONLINEAR OPERATOR APPROXIMATION THEORY
}

\author{
J. APPell AND M. P. PERA
}

\begin{abstract}
This paper, which builds on recent work of Anselone and Ansorge, is concerned with the approximate solution of nonlinear equations involving noncompact operators. Roughly speaking, the concepts developed (such as "measure of $d$-noncompactness", "generalized noncompact convergence", etc.) play the same role for approximation problems as the theory of condensing operators for existence problems.
\end{abstract}

1. Introduction. One of the fundamental concepts in nonlinear functional analysis is that of compactness. For example, the classical fixed point principles, degree theories, and bifurcation results are based on compactness arguments. Most of these results, however, involve nonconstructive existence proofs. The necessity of calculating (or at least approximating) solutions to integral or differential equations created a large literature on compact operator approximation. As an example, we mention the survey papers [3] and [4], which are essentially self-contained, and provide a large amount of methods, results, and examples, and were actually the motivation for the present paper: In fact, despite the importance of compactness principles, from the viewpoint of applications it seems worthwhile to extend the theory of operator approximation to a larger class of mappings. One of the simplest such classes is that of condensing mappings, i.e. those which diminish some measure of noncompactness. (A good recent survey on condensing operators is [1], on their application to functional-differential equations is [2].) It turns out that a suitable way of describing noncompact operator approximation is to introduce " $d$-condensing" mappings, i.e. those which diminish some measure of $d$-noncompactness (which means "lack of discrete compactness"). It is the purpose of this paper to develop this idea and to study its applicability to analytic problems, where noncompact operators occur.

It should be mentioned that there exists already some literature on noncompact approximation theory (e.g. on the links between condensing maps and Galerkin methods). In the present paper, however, we restrict ourselves to generalizing solely the concepts described in [4], but as systematically as possible. Unfortunately, this made it necessary to introduce a great number of numerical characteristics in $\$ \S 2$ and 3 , all 
describing the "lack of some regularity" (compactness, $d$-compactness, convergence etc.). At least in part these characteristics will be justified by a model example in $\S 4$; we request the reader's indulgence until then.

2. Discrete noncompactness of sets. Let $X$ be a real Banach space with norm \|\| . Let $\left[x_{n}\right]_{N}$ be any sequence of elements $x_{n} \in X(N$ being an infinite index set of natural numbers). We will always distinguish between sequences $\left[x_{n}\right]_{N}$ and their values $\left\{x_{n}\right\}=\left\{x_{n}: n \in N\right\}$. Let

$$
\left[x_{n}\right]_{N}^{*}=\left\{x: x \in X, \exists N^{\prime} \subseteq N \text { such that } x_{n} \rightarrow x\left(n \in N^{\prime}\right)\right\}
$$

denote the set of all accumulation points (limits of subsequences) of the sequence $\left[x_{n}\right]_{N}$. Similarly, for a sequence $\left[S_{n}\right]_{N}$ of subsets $S_{n} \subseteq X$ let

$$
\left[S_{n}\right]_{N}^{*}=\bigcup\left\{\left[x_{n}\right]_{N^{\prime}}^{*}: N^{\prime} \subseteq N, x_{n} \in S_{n} \text { for all } n \in N^{\prime}\right\} .
$$

Clearly, $\left[\left\{x_{n}\right\}\right]_{N}^{*}=\left[x_{n}\right]_{N}^{*}$.

In what follows, we shall sometimes omit the index $N$ when the context is clear.

The following properties are not hard to verify:

(2.1) $S_{n} \subseteq T_{n}$ for all $n$ implies $\left[S_{n}\right]^{*} \subseteq\left[T_{n}\right]^{*}$;

(2.2) $\left[S_{n}\right]^{*}$ is a closed subset of $X$;

(2.3) $\left[S_{n}\right]^{*} \subseteq \overline{U_{n} S_{n}}$.

Recall (see [4]) that a sequence $\left[S_{n}\right]_{N}$ is said to be $d$-compact (discretely compact) if $\left[S_{n}\right]_{N^{\prime}}^{*} \neq \varnothing$ for any $N^{\prime} \subseteq N$ for which $S_{n} \neq \varnothing$ for $n \in N^{\prime}$. Thus, in particular, $\left[x_{n}\right]_{N}$ is $d$-compact if and only if $\left[x_{n}\right]_{N}^{*} \neq \varnothing$.

For any $S \subseteq X$ and $\varepsilon \geq 0$ let

$$
\Omega_{\varepsilon}(S)=\bigcup_{x \in S} \bar{B}_{\varepsilon}(x),
$$

where $\bar{B}_{\varepsilon}(x)=\left\{x^{\prime}: x^{\prime} \in X,\left\|x^{\prime}-x\right\| \leq \varepsilon\right\} ;$ let $\Omega_{\varepsilon}(\varnothing)=\varnothing$.

Theorem 2.1 below contains two characterizations of the $d$-compactness of a sequence $\left[S_{n}\right]_{N}$. For proofs and further details we refer to [4].

THEOREM 2.1. Let $\left[S_{n}\right]_{N}$ be a sequence of subsets $S_{n} \subseteq X$. Then

(i) $\left[S_{n}\right]_{N}$ is d-compact if and only if $\left[S_{n}\right]_{N}^{*}$ is compact and for any $\varepsilon>0$ there exists $n_{\varepsilon} \in N$ such that $S_{n} \subseteq \Omega_{\varepsilon}\left(\left[S_{n}\right]_{N}^{*}\right)$ for all $n \geq n_{\varepsilon}$;

(ii) $\bar{U}_{n} S_{n}$ is compact if and only if $\left[S_{n}\right]_{N}$ is d-compact and the sets $\overline{S_{n}}$ are compact for each $n \in N$.

For a given $S \subseteq X$, let us recall the Hausdorff measure of noncompactness (see e.g. [11, 13])

$$
\alpha(S)=\inf \{\varepsilon: \varepsilon>0, \text { there exists a finite } \varepsilon \text {-net for } S\}
$$


with the properties:

(i) $\alpha(S)<\infty$ if and only if $S$ is bounded;

(ii) $\alpha(S)=0$ if and only if $\bar{S}$ is compact;

(iii) $S \subseteq T$ implies $\alpha(S) \leq \alpha(T)$;

(iv) $\alpha(S \cup T)=\max \{\alpha(S), \alpha(T)\}$;

(v) $\alpha(S \cap T) \leq \min \{\alpha(S), \alpha(T)\}$;

(vi) $\alpha(S+T) \leq \alpha(S)+\alpha(T)$;

(vii) $\alpha(\lambda S)=|\lambda| \alpha(S)$ for $\lambda \in \mathbf{R}$

(viii) $\alpha(\overline{\operatorname{co} S})=\alpha(S)$, where $\overline{\text { co }}$ denotes the closed convex hull.

After these preliminaries we are now in a position to introduce our measure of discrete noncompactness $\delta$. Namely, for a given sequence $\left[S_{n}\right]_{N}$ of bounded sets $S_{n} \subset X$ we define

$$
\delta\left(\left[S_{n}\right]_{N}\right)=\alpha\left(\left[S_{n}\right]_{N}^{*}\right)+\omega\left(\left[S_{n}\right]_{N}\right),
$$

where

$$
\begin{aligned}
& \omega\left(\left[S_{n}\right]_{N}\right)=\inf \left\{\varepsilon: \varepsilon>0, \text { there exists } n_{\varepsilon} \in N\right. \text { such that } \\
& \left.\qquad S_{n} \subseteq \Omega_{\varepsilon}\left(\left[S_{n}\right]_{N}^{*}\right) \text { for all } n \geq n_{\varepsilon}\right\} .
\end{aligned}
$$

In particular, for a given sequence $\left[x_{n}\right]_{N}$,

$$
\delta\left(\left[x_{n}\right]_{N}\right)=\alpha\left(\left[x_{n}\right]_{N}^{*}\right)+\omega\left(\left[x_{n}\right]_{N}\right)
$$

where

$$
\begin{aligned}
& \omega\left(\left[x_{n}\right]_{N}\right)=\inf \left\{\varepsilon: \varepsilon>0, \text { there exists } n_{\varepsilon} \in N\right. \text { such that } \\
& \left.\qquad \operatorname{dist}\left(x_{n},\left[x_{n}\right]_{N}^{*}\right) \leq \varepsilon \text { for all } n \geq n_{\varepsilon}\right\} .
\end{aligned}
$$

EXAMPLE 2.1. (a) For any $n \in N$, let $S_{n}$ be the unit ball $B_{1}(0)$. Then $\omega\left(\left[S_{n}\right]\right)=0$ and $\alpha\left(\left[S_{n}\right]^{*}\right)=\alpha\left(\widetilde{B}_{1}(0)\right)=1$, so $\delta\left(\left[S_{n}\right]\right)=1$.

(b) Let $X$ be a Hilbert space with countable $O N$-basis $\left\{e_{1}, e_{2}, e_{3}, \ldots\right\}$. Take $\left[x_{n}\right]=\left[0, e_{1}, 0, e_{2}, \ldots\right]$. Then $\alpha\left(\left[x_{n}\right]^{*}\right)=\alpha(\{0\})=0$ and $\delta\left(\left[x_{n}\right]\right)=$ $\omega\left(\left[x_{n}\right]\right)=1$.

Let us point out that neither $\omega$ nor $\delta$ is monotone. In fact, let $X$ be the Banach space of continuous functions over the interval $[0,1]$ and let $z_{n}$ be the characteristic function of the interval $\left[n^{-1},(n-1)^{-1}\right]$. Clearly, for $n \neq m$ we have $\left\|z_{n}-z_{m}\right\|=1$. Take $S_{n}=\left\{0, z_{1}, \ldots, z_{n}\right\}, T_{n}=\operatorname{co} S_{n}$. Then $\alpha\left(\left[S_{n}\right]^{*}\right)=0, \omega\left(\left[S_{n}\right]\right)=1$, and, since $\left[T_{n}\right]^{*}=\bigcup_{n} T_{n}, \alpha\left(\left[T_{n}\right]^{*}\right)=1 / 2$, $\omega\left(\left[T_{n}\right]\right)=0$. Thus $\delta\left(\left[S_{n}\right]\right)=1>1 / 2=\delta\left(\left[T_{n}\right]\right)$. 
One might suspect that there is at least some constant $c>0$ such that $\delta\left(\left[S_{n}\right]\right) \leq c \delta\left(\left[T_{n}\right]\right)$ whenever $S_{n} \subseteq T_{n}$. The following example shows that this is not so:

Consider an infinite dimensional Banach space $X$ and sequences $\left[y_{n}\right]$ and $\left[z_{n}\right]$ in $X$ such that $\left\|y_{n}\right\|=1,\left[y_{n}\right]^{*}=\varnothing,\left\|z_{n}-z_{0}\right\|=1\left(\left\|z_{0}\right\|>2\right)$, and $\left[z_{n}\right]^{*}=\varnothing$. Define $\left[x_{n}\right]=\left[0, y_{1}, z_{1}, 0, y_{2}, z_{2}, \ldots\right], S_{n}=$ $\left\{x_{1}, x_{2}, \ldots, x_{3 n}\right\}$, and $T_{n}=B_{1}\left(z_{0}\right) \cup \operatorname{co}\left\{0, y_{1}, \ldots, y_{n}\right\}$. Then $\left[S_{n}\right]^{*}=\{0\}$, $\left[T_{n}\right]^{*}=\bar{B}_{1}\left(z_{0}\right) \cup \overline{\operatorname{co}}\left\{0, y_{1}, y_{2}, \ldots\right\}$, so that $\alpha\left(\left[S_{n}\right]^{*}\right)=\omega\left(\left[T_{n}\right]\right)=0, \omega\left(\left[S_{n}\right]\right)$ $\geq\left\|z_{0}\right\|-1$, and $\alpha\left(\left[T_{n}\right]^{*}\right)=1$. Therefore, letting $\left\|z_{0}\right\| \rightarrow \infty$ we can make $\delta\left(\left[S_{n}\right]\right)=\omega\left(\left[S_{n}\right]\right)$ arbitrarily large, while $\delta\left(\left[T_{n}\right]\right)$ remains equal to 1 independently of $z_{0}$.

Theorem 2.1, interpreted in terms of $\alpha, \omega$ and $\delta$, reads as follows:

(2.4) $\left[S_{n}\right]_{N}$ is d-compact if and only if $\delta\left(\left[S_{n}\right]_{N}\right)=0$;

(2.5) $\alpha\left(\bigcup_{n} S_{n}\right)=0$ if and only if $\delta\left(\left[S_{n}\right]_{N}\right)=0$ and $\sup _{n} \alpha\left(S_{n}\right)=0$.

In particular, we deduce that

(2.6) $\delta\left(\left[S_{n}\right]_{N}\right)=0$ implies $\alpha\left(\left[S_{n}\right]_{N}^{*}\right)=0$;

(2.7) $\alpha\left(\bigcup_{n} S_{n}\right)=0$ implies $\omega\left(\left[S_{n}\right]_{N}\right)=0$.

It should be observed that the converse implications do not hold as Example 2.1 shows. Obviously, (2.6) can easily be derived from the inequality $\alpha\left(\left[S_{n}\right]^{*}\right) \leq \delta\left(\left[S_{n}\right]\right)$, which holds by definition. On the other hand, a similar estimate for (2.7) (i.e. $\left.\omega\left(\left[S_{n}\right]\right) \leq c \alpha\left(\bigcup_{n} S_{n}\right)\right)$ does not hold. In fact, let $\left[z_{n}\right]$ be a sequence in the closed ball $\bar{B}_{r}\left(x_{0}\right)\left(\left\|x_{0}\right\|>r\right)$ without accumulation points and let $\left[x_{n}\right]=\left[0, z_{1}, 0, z_{2}, \ldots\right]$. Then $\alpha\left(\left\{x_{n}\right\}\right) \leq r$ and $\left\|x_{0}\right\|-r \leq \omega\left(\left[x_{n}\right]\right) \leq\left\|x_{0}\right\|+r$, which implies $\omega\left(\left[x_{n}\right]\right)>c \alpha\left(\left\{x_{n}\right\}\right)$ for $\left\|x_{0}\right\| \geq(c+1) r$.

It is not hard to give a direct proof of (2.7). Taking into account this fact, a suitable extension of (2.5) turns out to be the following:

Proposition 2.1. The equivalence

$$
\alpha\left(\bigcup_{n} S_{n}\right)+\omega\left(\left[S_{n}\right]_{N}\right) \sim \sup _{n} \alpha\left(S_{n}\right)+\delta\left(\left[S_{n}\right]_{N}\right)
$$

holds, i.e. there exists $c, C>0$ such that

$$
\begin{aligned}
c\left\{\alpha\left(\bigcup_{n} S_{n}\right)+\omega\left(\left[S_{n}\right]\right)\right\} & \leq \sup _{n} \alpha\left(S_{n}\right)+\delta\left(\left[S_{n}\right]\right) \\
& \leq C\left\{\alpha\left(\bigcup_{n} S_{n}\right)+\omega\left(\left[S_{n}\right]\right)\right\} .
\end{aligned}
$$


Proof. Since $S_{n} \subseteq \cup_{n} S_{n}$ and $\left[S_{n}\right]^{*} \subseteq \bar{U}_{n} S_{n}$, we get

$$
\begin{aligned}
\sup _{n} \alpha\left(S_{n}\right)+\delta\left(\left[S_{n}\right]\right) & =\sup _{n} \alpha\left(S_{n}\right)+\alpha\left(\left[S_{n}\right]^{*}\right)+\omega\left(\left[S_{n}\right]\right) \\
& \leq 2 \alpha\left(\bigcup_{n} S_{n}\right)+\omega\left(\left[S_{n}\right]\right),
\end{aligned}
$$

that is $C=2$. To show the left inequality, take $\varepsilon>\sup _{n} \alpha\left(S_{n}\right)+\delta\left(\left[S_{n}\right]\right)$ (clearly, if this sum is infinite, there is nothing to prove). Hence, $\varepsilon>\omega\left(\left[S_{n}\right]\right)$ so $\left[S_{n}\right]^{*} \neq \varnothing$ and there exists $n_{\varepsilon} \in N$ such that $S_{n} \subseteq \Omega_{\varepsilon}\left(\left[S_{n}\right]^{*}\right)$ for $n \geq n_{\varepsilon}$. Therefore,

$$
\bigcup_{n \geq n_{\varepsilon}} S_{n} \subseteq \Omega_{\varepsilon}\left(\left[S_{n}\right]^{*}\right)
$$

On the other hand, we also have $\varepsilon>\alpha\left(\left[S_{n}\right]^{*}\right)$ which implies $\alpha\left(\Omega_{\varepsilon}\left(\left[S_{n}\right]^{*}\right)\right)$ $<2 \varepsilon$. Thus,

$$
\alpha\left(\bigcup_{n} S_{n}\right)=\max \left\{\alpha\left(\bigcup_{n<n_{\varepsilon}} S_{n}\right), \alpha\left(\bigcup_{n \geq n_{\varepsilon}} S_{n}\right)\right\}<2 \varepsilon .
$$

Consequently, $\alpha\left(\bigcup_{n} S_{n}\right)+\omega\left(\left[S_{n}\right]\right)<3 \varepsilon$ and we can take $c=1 / 3$.

The idea of measuring the "lack of $d$-compactness" of a sequence is not new. For instance, in [16] a discrete noncompactness measure was introduced which in its simplest version has the form

$$
\begin{aligned}
& \chi\left(\left[x_{n}\right]_{N}\right)=\inf \left\{\varepsilon: \varepsilon>0, \text { for any } N^{\prime} \subseteq N \text { there exist } N^{\prime \prime} \subseteq N^{\prime}\right. \\
& \text { and } \left.x \in X \text { such that }\left\|x_{n}-x\right\| \leq \varepsilon \text { for all } n \in N^{\prime \prime}\right\} .
\end{aligned}
$$

We close this section by comparing our measure $\delta$ with $\chi$.

Proposition 2.2. For any given sequence $\left[x_{n}\right]_{N}$ in $X$ we have

(i) $\chi\left(\left[x_{n}\right]_{N}\right) \leq \delta\left(\left[x_{n}\right]_{N}\right)$;

(ii) $\alpha\left(\left[x_{n}\right]_{N}^{*}\right) \leq c \chi\left(\left[x_{n}\right]_{N}\right)$ for some $c>0$;

(iii) $\omega\left(\left[x_{n}\right]_{N}\right) \nless C \chi\left(\left[x_{n}\right]_{N}\right)$ for any $C>0$.

Proof. (i) If $\delta\left(\left[x_{n}\right]\right)$ is infinite, the assertion is trivial. So, let $\varepsilon>\delta\left(\left[x_{n}\right]\right)$ and take $\alpha>\alpha\left(\left[x_{n}\right]^{*}\right), \omega>\omega\left(\left[x_{n}\right]\right)$ such that $\delta\left(\left[x_{n}\right]\right)<\alpha+\omega \leq \varepsilon$. Hence $\left[x_{n}\right]^{*} \neq \varnothing$ and there exists $n_{\omega} \in N$ such that $x_{n} \in \Omega_{\omega}\left(\left[x_{n}\right]^{*}\right)$ for $n \geq n_{\omega}$. Consequently, for any $n \geq n_{\omega}$, we have $\left\|x_{n}-y_{n}\right\| \leq \omega$ for some $y_{n} \in\left[x_{n}\right]^{*}$. On the other hand, there exists a finite $\alpha$-net in $X$, say $\left\{z_{1}, \ldots, z_{m}\right\}$, such that $\left[x_{n}\right]^{*} \subseteq B_{\alpha}\left(z_{1}\right) \cup B_{\alpha}\left(z_{2}\right) \cup \cdots \cup B_{\alpha}\left(z_{m}\right)$. Thus, if $\left[x_{n}\right]_{N^{\prime}}\left(N^{\prime} \subseteq N\right)$ 
is any subsequence of $\left[x_{n}\right]_{N^{\prime}}$ one can find an index $j \in\{1, \ldots, m\}$ and an infinite subset $N^{\prime \prime}$ of $N^{\prime}$ such that $y_{n} \in B_{\alpha}\left(z_{J}\right)$ for $n \in N^{\prime \prime}$. So, for $n \in N^{\prime \prime}$,

$$
\left\|x_{n}-z_{j}\right\| \leq\left\|x_{n}-y_{n}\right\|+\left\|y_{n}-z_{j}\right\| \leq \omega+\alpha \leq \varepsilon,
$$

which implies $\chi\left(\left[x_{n}\right]\right) \leq \varepsilon$.

(ii) As above, we may restrict ourselves to the case when $\chi\left(\left[x_{n}\right]\right)$ is finite. Let $\varepsilon>\chi\left(\left[x_{n}\right]\right)$ and suppose $\alpha\left(\left[x_{n}\right]^{*}\right)>3 \varepsilon$. There exists a sequence $\left[y_{m}\right]$ in $\left[x_{n}\right]^{*}$ such that $\left\|y_{p}-y_{q}\right\|>3 \varepsilon$ for $p \neq q$. On the other hand, for any $m$, the sequence $\left[x_{n}\right]_{N}$ has a subsequence $\left[x_{n}^{(m)}\right]_{N^{\prime}}\left(N^{\prime} \subseteq N\right)$ converging to $y_{m}$. Take the diagonal sequence $\left[x_{n}^{(n)}\right]_{N^{\prime}}$. Clearly, $\left\|x_{n}^{(n)}-y_{n}\right\| \leq$ $\varepsilon_{n} \rightarrow 0\left(n \in N^{\prime}\right)$. Observe also that $\left[x_{n}^{(n)}\right]_{N^{\prime}}$ is a subsequence of $\left[x_{n}\right]_{N}$. So, since $\varepsilon>\chi\left(\left[x_{n}\right]\right)$, there exist $N^{\prime \prime} \subseteq N^{\prime}$ and $x \in X$ such that $\left\|x_{n}^{(n)}-x\right\| \leq \varepsilon$ ( $\left.n \in N^{\prime \prime}\right)$. Thus, for $p, q$ sufficiently large, we get

$$
\begin{aligned}
3 \varepsilon & <\left\|y_{p}-y_{q}\right\| \leq\left\|y_{p}-x_{p}^{(p)}\right\|+\left\|x_{p}^{(p)}-x\right\|+\left\|x-x_{q}^{(q)}\right\|+\left\|x_{q}^{(q)}-y_{q}\right\| \\
& \leq \varepsilon_{p}+2 \varepsilon+\varepsilon_{q} \leq 3 \varepsilon,
\end{aligned}
$$

a contradiction. Therefore $\alpha\left(\left[x_{n}\right]^{*}\right) \leq 3 \varepsilon$ and assertion (ii) is proved with $c=3$.

(iii) We give a counterexample. Let $H$ be a hyperplane in an infinite dimensional space $X$, i.e. $X=H \oplus \mathbf{R} x_{0}, x_{0} \neq 0$. Let $\left[z_{n}\right]$ be a sequence in $H$ such that $\left\|z_{n}\right\|=1$ and $\left[z_{n}\right]^{*}=\varnothing$. Consider $\left[x_{n}\right]=$ $\left[z_{1}, \lambda x_{0}, z_{2}, \lambda x_{0}, z_{3}, \ldots\right], \lambda>0$. It is easily seen that $\chi\left(\left[x_{n}\right]\right)=1$. Moreover, since $\left[x_{n}\right]^{*}=\left\{\lambda x_{0}\right\}$, we have $\omega\left(\left[x_{n}\right]\right) \geq \lambda\left\|x_{0}\right\|$. Therefore $\omega\left(\left[x_{n}\right]\right)>$ $C \chi\left(\left[x_{n}\right]\right)$ for $\lambda>C /\left\|x_{0}\right\|$.

It is clear from the above result that $\chi$ and $\delta$ are not linearly equivalent. However, both are measures of $d$-noncompactness (in the sense that they are zero exactly on $d$-compact sequences), while $\delta$ seems to be "coarser" in some sense than $\chi$ (because of (iii)).

3. Discrete noncompactness of operators. Let $K$ and $K_{n}(n \in N)$ be (not necessarily linear) operators acting between two Banach spaces $X$ and $Y$. Recall the following definitions (see e.g. [4]):

$K$ is called compact if $\overline{K(S)}$ is compact for any bounded $S \subset X$; the sequence $\left[K_{n}\right]_{N}$ is called collectively compact if $\overline{U_{n} K_{n}(S)}$ is compact, and asymptotically compact (or $d$-compact) if $\left[K_{n}(S)\right]_{N}$ is $d$-compact for any bounded $S \subset X$.

The following relation between these notions is shown in [4]:

THEOREM 3.1. $\left[K_{n}\right]_{N}$ is collectively compact if and only if $\left[K_{n}\right]_{N}$ is asymptotically compact and $K_{n}$ is compact for each $n \in N$. 
Let us now introduce some further parameters (for the definition of $\alpha\left(\left[K_{n}\right]_{N}\right)$ see also $\left.[10,17]\right)$ :

$$
\begin{gathered}
\alpha(K)=\inf \{\lambda: \lambda>0, \alpha(K(S)) \leq \alpha(S), S \subset X \text { bounded }\} ; \\
\alpha\left(\left[K_{n}\right]_{N}\right)=\inf \left\{\lambda: \lambda>0, \alpha\left(\bigcup_{n} K_{n}(S)\right) \leq \lambda \alpha(S), S \subset X \text { bounded }\right\} ; \\
\omega\left(\left[K_{n}\right]_{N}\right)=\inf \left\{\lambda: \lambda>0, \omega\left(\left[K_{n}(S)\right]_{N}\right) \leq \lambda \alpha(S), S \subset X \text { bounded }\right\} ; \\
\delta\left(\left[K_{n}\right]_{N}\right)=\inf \left\{\lambda: \lambda>0, \delta\left(\left[K_{n}(S)\right]_{N}\right) \leq \lambda \alpha(S), S \subset X \text { bounded }\right\} .
\end{gathered}
$$

According to the above definitions, we have:

(3.1) $\alpha(K)=0$ if and only if $K$ is compact;

(3.2) $\alpha\left(\left[K_{n}\right]\right)=0$ if and only if $\left[K_{n}\right]$ is collectively compact;

(3.3) $\delta\left(\left[K_{n}\right]\right)=0$ if and only if $\left[K_{n}\right]$ is asymptotically compact;

(3.4) $\alpha\left(\left[K_{n}\right]\right)=0$ implies $\omega\left(\left[K_{n}\right]\right)=0$.

Moreover, from Proposition 2.1 we can immediately deduce the following extension of Theorem 3.1:

Proposition 3.1. The equivalence

$$
\alpha\left(\left[K_{n}\right]_{N}\right)+\omega\left(\left[K_{n}\right]_{N}\right) \sim \sup _{n} \alpha\left(K_{n}\right)+\delta\left(\left[K_{n}\right]_{N}\right)
$$

holds, where $\sim$ is defined as in Proposition 2.1.

The notions of compact, collectively compact, and asymptotically compact operators can be characterized also in terms of sequences of elements in $X$ (see [4]). From this point of view, in our context we are led to

Proposition 3.2. Let $\left[x_{n}\right]_{N}$ be a bounded sequence in $X$, and let $\pi: N \rightarrow N$ be any bijection. Then

(i) $\delta\left(\left[K x_{n}\right]_{N}\right) \leq \alpha(K) \alpha\left(\left\{x_{n}\right\}\right)+\omega\left(\left[K x_{n}\right]_{N}\right)$;

(ii) $\delta\left(\left[K_{\pi(n)} x_{n}\right]_{N}\right) \leq \alpha\left(\left[K_{n}\right]_{N}\right) \alpha\left(\left\{x_{n}\right\}\right)+\omega\left(\left[K_{\pi(n)} x_{n}\right]\right)$;

(iii) $\delta\left(\left[K_{n} x_{n}\right]_{N}\right) \leq \delta\left(\left[K_{n}\right]_{N}\right) \alpha\left(\left\{x_{n}\right\}\right)$.

In particular,

(i) $\alpha(K)=0$ if and only if $\delta\left(\left[K x_{n}\right]_{N}\right)=0$ for any bounded sequence $\left[x_{n}\right]_{N}$

(ii) $^{\prime} \alpha\left(\left[K_{n}\right]_{N}\right)=0$ if and only if $\delta\left(\left[K_{\pi(n)} x_{n}\right]_{N}\right)=0$ for any bounded sequence $\left[x_{n}\right]_{N}$ and bijection $\pi$;

(iii) $^{\prime} \delta\left(\left[K_{n}\right]_{N}\right)=0$ if and only if $\delta\left(\left[K_{n} x_{n}\right]_{N}\right)=0$ for any bounded sequence $\left[x_{n}\right]_{N}$. 
Proof. It suffices to observe that for any $\varepsilon>0$,

$$
\begin{aligned}
\alpha\left(\left[K x_{n}\right]^{*}\right) & \leq \alpha\left(\left\{K x_{n}\right\}\right) \leq(\alpha(K)+\varepsilon) \alpha\left(\left\{x_{n}\right\}\right), \\
\alpha\left(\left[K_{\pi(n)} x_{n}\right]^{*}\right) & \leq \alpha\left(\left\{K_{\pi(n)} x_{n}\right\}\right) \leq\left(\alpha\left(\left[K_{n}\right]\right)+\varepsilon\right) \alpha\left(\left\{x_{n}\right\}\right), \\
\delta\left(\left[K_{n} x_{n}\right]\right) & \leq\left(\delta\left(\left[K_{n}\right]\right)+\varepsilon\right) \alpha\left(\left\{x_{n}\right\}\right),
\end{aligned}
$$

and moreover, that

$$
\begin{array}{ccc}
\alpha(K)=0 & \text { implies } & \omega\left(\left[K x_{n}\right]\right)=0, \\
\alpha\left(\left[K_{n}\right]\right)=0 & \text { implies } & \omega\left(\left[K_{\pi(n)} x_{n}\right]\right)=0 .
\end{array}
$$

We will now introduce various types of convergence for operator sequences.

$$
\begin{aligned}
K_{n} \rightarrow K \quad & \text { (pointwise convergence) means that } K_{n} x \rightarrow K x \\
& \text { as } n \rightarrow \infty \text { for all } x \in X ; \\
K_{n} \stackrel{c}{\rightarrow} K \quad & \text { (continuous convergence) means that } x \rightarrow x_{0} \\
& \text { implies } K_{n} x \rightarrow K x_{0}(n \rightarrow \infty), \text { or equivalently } \\
& x_{n} \rightarrow x_{0}(n \rightarrow \infty) \text { implies } K_{n} x_{n} \rightarrow K x_{0}(n \rightarrow \infty), \\
& \text { or equivalently } x_{m} \rightarrow x_{0}(m \rightarrow \infty) \text { implies } \\
& K_{n} x_{m} \rightarrow K x_{0}(m, n \rightarrow \infty) .
\end{aligned}
$$

Observe that $K_{n} \stackrel{c}{\rightarrow} K$ implies the continuity of $K$; furthermore, for continuous $K_{n}$ the continuous convergence $K_{n} \stackrel{c}{\rightarrow} K$ is equivalent to the pointwise convergence $K_{n} \rightarrow K$ and to the equicontinuity of the family [ $\left.K_{n}\right]$. Moreover, for linear continuous $K, K_{n}$ from the Banach-Steinhaus theorem it follows that pointwise convergence implies equicontinuity, and hence pointwise and continuous convergence coincide.

Furthermore, we will define two other types of convergence, generalizing those in [4]; given $\lambda \geq 0$,

$K_{n} \stackrel{\lambda \text {-cc }}{\rightarrow} K \quad(\lambda$-collectively compact convergence $)$ means

that each $K_{n}$ is continuous, $K_{n} \stackrel{c}{\rightarrow} K$, and

$\alpha\left(\left[K_{n}\right]_{N}\right)+\omega\left(\left[K_{n}\right]_{N}\right) \leq \lambda ;$

$K_{n} \stackrel{\lambda \text {-ac }}{\rightarrow} K \quad(\lambda$-asymptotically compact convergence $)$ means

that each $K_{n}$ is continuous, $K_{n} \stackrel{c}{\rightarrow} K$, and

$\delta\left(\left[K_{n}\right]_{N}\right) \leq \lambda$. 
We remark that, for $\lambda=0$, our 0 -cc and 0 -ac convergences are nothing else but the cc and ac convergences introduced in [4]. Hence, the next proposition generalizes corresponding results in [4].

Proposition 3.3. The following relations hold:

(i) $K_{n} \rightarrow K$ implies $\alpha(K) \leq \alpha\left(\left[K_{n}\right]_{N}\right)$;

(ii) $K_{n} \stackrel{c}{\rightarrow} K$ implies $\alpha(K) \leq \delta\left(\left[K_{n}\right]_{N}\right)$;

(iii) $K_{n} \stackrel{\lambda \text {-cc }}{\rightarrow} K$ implies $K_{n} \stackrel{\lambda \text {-ac }}{\rightarrow} K$ and $\sup _{n} \alpha\left(K_{n}\right) \leq \lambda$;

(iv) $K_{n} \stackrel{\lambda \text {-ac }}{\rightarrow} K$ and $\sup _{n} \alpha\left(K_{n}\right) \leq \lambda$ imply $K_{n} \stackrel{C \lambda \text {-cc }}{\rightarrow} K$ for some $C>0$.

Proof. To show (i), (ii) and (iii) it suffices to observe that, for any bounded subset $S \subset X$, we have

$$
\overline{K(S)} \subseteq\left[K_{n}(S)\right]_{N}^{*} \subseteq \overline{\bigcup_{n} K_{n}(S)}
$$

(iv) follows from Proposition 3.1.

Let us now consider $X=Y$ and $K, K_{n}$ as operators in $X$. Our aim is to obtain information about the nonemptiness of the set

$$
\operatorname{Fix}(K, S)=\{x: x \in S \subseteq X, K x=x\}
$$

and especially of $\operatorname{Fix}(K)=\operatorname{Fix}(K, X)$. This will be carried out by means of the various kinds of convergence introduced above, more precisely by comparing the fixed point problem $K x=x$ with the exact problem $K_{n} x=x$ or with the approximate problem $\left\|K_{n} x_{n}-x_{n}\right\| \rightarrow 0$.

Lemma 3.1 below will be used in proving our main results.

LEMMA 3.1. The following relations hold:

(i) If $\alpha(K)<1$, then $\alpha(\operatorname{Fix}(K, S))=0$ for any bounded $S \subset X$.

(ii) Let $\left[S_{n}\right]_{N}$ be any sequence of subsets in $X$ such that $\cup_{n} S_{n}$ is bounded. Then $\left[S_{n}\right]_{N}^{*} \neq \varnothing$ if and only if $\delta\left(\left[S_{n}\right]_{N}\right)<\infty$.

(iii) Let $\left[S_{n}\right]_{N},\left[T_{n}\right]_{N}$ be sequences of subsets in $X$. Let $\Delta(S, T)$ be the Hausdorff distance of $S$ and $T$, i.e. the infimum of all $\varepsilon>0$ such that $S \subseteq \Omega_{\varepsilon}(T)$ and $T \subseteq \Omega_{\varepsilon}(S)$. Then $\Delta\left(S_{n}, T_{n}\right) \rightarrow 0(n \rightarrow \infty)$ implies $\delta\left(\left[S_{n}\right]_{N}\right)$ $=\delta\left(\left[T_{n}\right]_{N}\right)$.

Proof. There exists $\lambda>0$ such that $\alpha(K)<\lambda<1$. On the other hand, since $\operatorname{Fix}(K, S)=K(\operatorname{Fix}(K, S))$, we have

$$
\alpha(\operatorname{Fix}(K, S))=\alpha(K(\operatorname{Fix}(K, S))) \leq \lambda \alpha(\operatorname{Fix}(K, S)) .
$$

Thus, $\alpha(\operatorname{Fix}(K, S))=0$. 
To prove (ii), let $r>0$ be such that $\alpha\left(\left[S_{n}\right]^{*}\right) \leq \alpha\left(\cup_{n} S_{n}\right) \leq r$ and let $x \in\left[S_{n}\right]^{*}$. For any $n \in N, S_{n} \subseteq \bar{B}_{r}(0)$. Hence, $S_{n} \subseteq \Omega_{\varepsilon}\left(\left[S_{n}\right]^{*}\right)$, for $\varepsilon>$ $\|x\|+r$. Thus, also $\omega\left(\left[S_{n}\right]\right)$ is finite. Conversely, if $\left[S_{n}\right]^{*}=\varnothing$ then, by definition, $\omega\left(\left[S_{n}\right]\right)=\infty$.

Under the assumptions of (iii), let us show first that $\left[S_{n}\right]^{*}=\left[T_{n}\right]^{*}$. Take $x \in\left[S_{n}\right]^{*}$ and, for each $n \in N, x_{n} \in S_{n}$ such that $x_{n} \rightarrow x$. Now, for any $\varepsilon>0$ there exists $n_{\varepsilon} \in N$ such that $S_{n} \subseteq \Omega_{\varepsilon}\left(T_{n}\right), T_{n} \subseteq \Omega_{\varepsilon}\left(S_{n}\right)$ for all $n \geq n_{\varepsilon}$. Therefore, for any $n \geq n_{\varepsilon}$ one can find $y_{n} \in T_{n}$ such that $\left\|x_{n}-y_{n}\right\|$ $<\varepsilon$. Thus, $y_{n} \rightarrow x$ and, consequently, $x \in\left[T_{n}\right]^{*}$. Analogously, $\left[T_{n}\right]^{*} \subseteq$ $\left[S_{n}\right]^{*}$. Observe now that the equality $\left[S_{n}\right]^{*}=\left[T_{n}\right]^{*}$ implies $\omega\left(\left[S_{n}\right]\right)<\infty$ if and only if $\omega\left(\left[T_{n}\right]\right)<\infty$. In fact, suppose $\omega\left(\left[S_{n}\right]\right)<\infty$. Then there exists $\omega>\omega\left(\left[S_{n}\right]\right)$ and $n_{\omega} \in N$ such that, for all $n \geq n_{\omega}$,

$$
S_{n} \subseteq \Omega_{\omega}\left(\left[S_{n}\right]^{*}\right)=\Omega_{\omega}\left(\left[T_{n}\right]^{*}\right) .
$$

Moreover, for any $\varepsilon>0$ there exists $n_{\varepsilon} \in N$ such that, for $n \geq n_{\varepsilon}$, $T_{n} \subseteq \Omega_{\varepsilon}\left(S_{n}\right)$. Consequently, for $n \geq \max \left\{n_{\omega}, n_{\varepsilon}\right\}$, we have $T_{n} \subseteq$ $\Omega_{\omega+\varepsilon}\left(\left[T_{n}\right]^{*}\right)$, so $\omega\left(\left[T_{n}\right]\right) \leq \omega+\varepsilon$. Since this inequality holds for all $\varepsilon>0$, we have also proved that $\omega\left(\left[T_{n}\right]\right) \leq \omega\left(\left[S_{n}\right]\right)$. By symmetry we clearly obtain the converse. Consequently, $\delta\left(\left[S_{n}\right]\right)=\delta\left(\left[T_{n}\right]\right)$ as claimed.

We state now some existence and convergence results which contain, as particular cases, Theorems 3.7 and 3.8 of [4].

Theorem 3.2. Let $K_{n}, K$ be operators in $X$. Suppose there exists a sequence $\left[x_{n}\right]_{N}$ in $X$ such that $\left\|K_{n} x_{n}-x_{n}\right\| \rightarrow 0$. Then

(i) $K_{n} \stackrel{c}{\rightarrow} K$ implies

$$
\delta\left(\left[K_{n} x_{n}\right]_{N}\right)=\delta\left(\left[x_{n}\right]_{N}\right) \text { and }\left[x_{n}\right]_{N}^{*} \subseteq \operatorname{Fix}(K) ;
$$

(ii) If $\left[x_{n}\right]_{N}$ is bounded, then

$$
\delta\left(\left[x_{n}\right]_{N}\right) \leq \delta\left(\left[K_{n}\right]_{N}\right) \alpha\left(\left\{x_{n}\right\}\right)
$$

and

$$
\delta\left(\left[x_{n}\right]_{N}\right) \leq\left(\alpha\left(\left[K_{n}\right]_{N}\right)+\omega\left(\left[K_{n}\right]_{N}\right)\right) \alpha\left(\left\{x_{n}\right\}\right) ;
$$

(iii) If $K_{n} \stackrel{\lambda \text {-ac }}{\rightarrow} K$ and $\left[x_{n}\right]_{N}$ is bounded, then

$$
\delta\left(\left[x_{n}\right]_{N}\right) \leq \lambda \alpha\left(\left\{x_{n}\right\}\right) \text { and } \varnothing \neq\left[x_{n}\right]_{N}^{*} \subseteq \operatorname{Fix}(K) .
$$

Proof. (i) By (iii) of Lemma 3.1, and since $\left[K_{n} x_{n}-x_{n}\right]$ converges to zero, we immediately get $\delta\left(\left[K_{n} x_{n}\right]\right)=\delta\left(\left[x_{n}\right]\right)$. Moreover, if $x \in\left[x_{n}\right]^{*}$, 
there exists $N^{\prime} \subseteq N$ such that $x_{n} \rightarrow x\left(n \in N^{\prime}\right)$. Therefore,

$$
\|K x-x\| \leq\left\|K x-K_{n} x_{n}\right\|+\left\|K_{n} x_{n}-x_{n}\right\|+\left\|x_{n}-x\right\|
$$

and the right-hand side converges to zero as $n \rightarrow \infty$.

(ii) As above, $\delta\left(\left[K_{n} x_{n}\right]\right)=\delta\left(\left[x_{n}\right]\right)$. Thus, the assertion follows from Proposition 3.2 (iii) and (ii).

(iii) As above, $\delta\left(\left[K_{n} x_{n}\right]\right)=\delta\left(\left[x_{n}\right]\right)$. Thus, $\delta\left(\left[x_{n}\right]\right) \leq \delta\left(\left[K_{n}\right]\right) \alpha\left(\left\{x_{n}\right\}\right)$ $\leq \lambda \alpha\left(\left\{x_{n}\right\}\right)$. This implies, in particular, $\delta\left(\left[x_{n}\right]\right)<\infty$. So, by (ii) of Lemma 3.1, we get $\left[x_{n}\right]^{*} \neq \varnothing$. The assertion follows now as in (i).

Observe that in (i) above, $\left[x_{n}\right]^{*}$ may be empty even if $\left[x_{n}\right]$ is bounded. In other words, (iii) is an existence result for fixed points, while (i) is not.

To give an example, let $\left[x_{n}\right]$ be a sequence in an infinite dimensional space $X$ such that $\left\|x_{n}\right\|=1$ for each $n \in N$ and $\left[x_{n}\right]^{*}=\varnothing$. Define $K_{n} x=(1+1 / n) x, K x=x$. Then $\left[K_{n} x_{n}-x_{n}\right]$ converges to zero and, if $x \rightarrow x_{0}$, one has

$$
\left\|K_{n} x-K x_{0}\right\|=\left\|\left(1+\frac{1}{n}\right) x-x_{0}\right\| \leq\left\|x-x_{0}\right\|+\frac{1}{n}\|x\| \rightarrow 0,
$$

which means $K_{n} \stackrel{c}{\rightarrow} K$. Clearly, $K_{n} \stackrel{\lambda \text {-ac }}{\rightarrow} K$ for any value of $\lambda$.

THEOREM 3.3. Let $K_{n}, K$ be operators in $X$ with $K_{n} \stackrel{\lambda \text {-ac }}{\rightarrow} K$. Let $\left[S_{n}\right]_{N}$ be a sequence of subsets in $X$ such that $\bigcup_{n} S_{n}$ is bounded, and let $0<\varepsilon_{n} \rightarrow 0$. Define

$$
\tilde{S}_{n}=\left\{x: x \in S_{n},\left\|x-K_{n} x\right\| \leq \varepsilon_{n}\right\} .
$$

Then

(i) $\delta\left(\left[\tilde{S}_{n}\right]_{N}\right) \leq \lambda \sup _{n} \alpha\left(\tilde{S}_{n}\right)$;

(ii) $\varnothing \neq\left[\tilde{S}_{n}\right]_{N}^{*} \subseteq \operatorname{Fix}(K, S)$ for any bounded $S \supseteq\left[S_{n}\right]_{N}^{*}$;

(iii) for any $\varepsilon>0$ there exists $n_{\varepsilon} \in N$ such that $n \geq n_{\varepsilon}$ implies $\tilde{S}_{n} \subseteq$ $\Omega_{\eta+\varepsilon}(\operatorname{Fix}(K, S))$ for any bounded $S \supseteq\left[S_{n}\right]_{N}^{*}$, where $\eta=\lambda \sup _{n} \alpha\left(\tilde{S}_{n}\right)$.

Proof. (i) Since $\Delta\left(\tilde{S}_{n}, K_{n}\left(\tilde{S}_{n}\right)\right) \leq \varepsilon_{n} \rightarrow 0$, from Lemma 3.1 (iii) we get $\delta\left(\left[\tilde{S}_{n}\right]\right)=\delta\left(\left[K_{n}\left(\tilde{S}_{n}\right)\right]\right)$. Hence, because of $\lambda$-ac convergence, $\delta\left(\left[\tilde{S}_{n}\right]\right) \leq$ $\lambda \alpha\left(\tilde{S}_{n}\right)$ for all $n \in N$.

(ii) By (i), $\delta\left(\left[\tilde{S}_{n}\right]\right)<\infty$. So, because of Lemma 3.1 (ii), $\left[\tilde{S}_{n}\right]^{*} \neq \varnothing$. Now apply Theorem 3.2 (i).

(iii) From (ii) we know that $\left[\tilde{S}_{n}\right]^{*} \subseteq \operatorname{Fix}(K, S)$. On the other hand, (i) implies that, given $\varepsilon>0$, there exists $n_{\varepsilon} \in N$ such that $\tilde{S}_{n} \subseteq \Omega_{\eta+\varepsilon}\left(\left[\tilde{S}_{n}\right]^{*}\right)$ for $n \geq n_{\varepsilon}$. 
4. Applications. In this section we shall illustrate Theorem 3.2 by means of two examples: First, we will study the significance of the assumptions of this theorem for a simple nonlinear operator, namely the Hammerstein operator

$$
K x(s)=\int_{\Omega} h(s, t) f(t, x(t)) d t
$$

in various function spaces. Second, we will indicate an application of Theorem 3.2 to an initial value problem for ordinary differential equations in a Banach space.

EXAMPLE 4.1. (a) Let $\Omega$ be a bounded domain in $\mathbf{R}^{N}$, and let $f_{n}, f$ and $h_{n}, h$ be real continuous functions on $\bar{\Omega} \times \mathbf{R}$ and $\bar{\Omega} \times \bar{\Omega}$, respectively. Consider the operators

$$
\begin{gathered}
H_{n} x(s)=\int_{\Omega} h_{n}(s, t) x(t) d t, \\
H x(s)=\int_{\Omega} h(s, t) x(t) d t,
\end{gathered}
$$

$$
F_{n} x(t)=f_{n}(t, x(t)), \quad F x(t)=f(t, x(t)) .
$$

Obviously, all these operators act in the space $X=C(\bar{\Omega})$ of continuous functions on $\bar{\Omega}$. Together with the operator (4.1) (i.e. $K x=H F x$ ) we consider the approximations

$$
K_{n} x(s)=H_{n} F_{n} x(s)=\int_{\Omega} h_{n}(s, t) f_{n}(t, x(t)) d t .
$$

Now, $K_{n} \stackrel{c}{\rightarrow} K$ means that uniform convergence (on $\bar{\Omega}$ ) of $\left[x_{n}\right]_{N}$ implies the same convergence of $\left[H_{n} F_{n} x_{n}\right]_{N}$. If this holds, then

$$
\left[K_{n}(S)\right]^{*}=K(S)
$$

for each bounded $S \subset X$. In fact, given $y \in\left[K_{n}(S)\right]^{*}$, there exist $N^{\prime} \subseteq N$ and a sequence $\left[x_{n}\right]$ in $S$ such that $K_{n} x_{n} \rightarrow y\left(n \in N^{\prime}\right)$. But since also $K_{n} x_{m} \rightarrow K x_{m}$ ( $m$ fixed), we must have $y=K x_{m}=K x_{m+1}=\cdots$ for some $m$, that is $y \in K(S)$. Conversely, given $y=K x(x \in S)$, take $y_{n}=K_{n} x$; obviously, $y_{n} \in K_{n}(S)$ and $y_{n} \rightarrow y$, so $y \in\left[K_{n}(S)\right]^{*}$.

As a consequence of $(4.5), \omega\left(\left[K_{n}(S)\right]\right)=0$. In other words, the condition $\delta\left(\left[K_{n}\right]\right) \leq \lambda$ (which is the condition $K_{n} \stackrel{\lambda \text {-ac }}{\rightarrow} K$ in Theorem 3.2 (iii)) means nothing else than

$$
\alpha(K) \leq \lambda
$$


Roughly speaking, this shows that for a $c$-convergent sequence $K_{n} \stackrel{c}{\rightarrow} K$ the discrete noncompactness of $\left[K_{n}\right]$ is essentially the noncompactness of $K$ (and vice versa).

Clearly, $\alpha(K) \leq\|H\| \alpha(F)$; but $\|H\|$ can be evaluated in terms of the kernel function $h$, and $\alpha(F)$ is exactly the minimal Lipschitz constant for the function $f$ with respect to the second argument (see [5, Theorem 1]).

(b) Consider now the same problem, but with $f_{n}, f$ satisfying only a Carathéodory condition. More precisely, let $F_{n}$ and $F$ map the space $L_{p}(\Omega)$ into the space $L_{q}(\Omega)(1 \leq q<p<\infty)$, while $H_{n}$ and $H$ vice versa. In this situation, $K_{n} \stackrel{c}{\rightarrow} K$ means that convergence in the mean (to the power $p$ ) of $\left[x_{n}\right]_{N}$ implies the same convergence of $\left[H_{n} F_{n} x_{n}\right]_{N}$. Again, we are led to the estimate (4.6). While $\|H\|$ can be evaluated in terms of the kernel function $h$ as before, the number $\alpha(F)$ is now given by

$$
\alpha(F)=c^{(p-q) / p}[q /(p-q)]^{-1 / p}[p /(p-q)]^{1 / q},
$$

where $c=\sup _{\varepsilon>0}\left\|a_{\varepsilon}\right\|_{q} \varepsilon^{q /(p-q)}<\infty$ and $f$ is supposed to satisfy a family of Lipschitz conditions

$$
|f(s, u)-f(s, v)| \leq a_{\varepsilon}(s)+\varepsilon|u-v|^{p / q} \quad\left(a_{\varepsilon} \in L_{q}(\Omega), \varepsilon>0\right),
$$

(see [5, Theorem 3]).

(c) Before considering the third variant of this example, we emphasize that all definitions and results obviously carry over to the case where the space $X$ is a metric space rather than a Banach space. (In fact, all constructions in [4] are posed in metric space settings.) So, let $X=S(\Omega)$ be the space of all (Lebesgue) measurable functions on $\Omega$, equipped with the metric

$$
d(x, y)=\inf _{h>0}\{h+\operatorname{meas}\{s: s \in \Omega,|x(s)-y(s)|>h\}\} ;
$$

convergence with respect to this metric coincides with convergence in measure. Given $f_{n}$ and $f$ on $\Omega \times \mathbf{R}$, it is known that (Lebesgue) measurability of these functions is neither sufficient ([14]) nor necessary ([8]) for the operators (4.3) to map the space $X$ into itself. Apart from the classical Carathéodory condition, there are many other sufficient conditions guaranteeing $F(X) \subseteq X$ (see e.g. [9] and the bibliography therein). Moreover, a condition on $f_{n}$ and $f$ which ensures that $x_{m} \rightarrow x$ (in measure) implies $F_{n} x_{m} \rightarrow F x$ (in measure) can be found in [15].

Concerning (4.6) in the space $X=S(\Omega)$, this condition follows certainly from a Lipschitz condition for $f$, but this is far from being necessary. Actually, we do not know conditions on the functions $f$ and $h$ which are both necessary and sufficient for (4.6). 
EXAMPLE 4.2. Let us now consider a more concrete example which shows the applicability of Theorem 3.2. To this end, consider in a Banach space $E$ the initial value problem for $t \in[0, T]$,

$$
x^{\prime}(t)=f(t, x(t)), \quad x(0)=x_{0} \in E,
$$

where $f$ is supposed to be uniformly continuous and bounded on $[0, T] \times$ $\bar{B}_{R}\left(x_{0}\right)$ with

$$
M=\sup \left\{\|f(t, x)\|_{E}: 0 \leq t \leq T,\left\|x-x_{0}\right\|_{E} \leq R\right\} \leq R / T .
$$

It is a well-known fact that these conditions, however, are not sufficient for local solvability of (4.7), but there must be some compactness condition in addition. As a typical existence theorem we mention the following result (see [1, p. 229], see also [12]):

THEOREM 4.1. Let $\alpha_{E}$ be the Hausdorff measure of noncompactness in the space E. Suppose

$$
\alpha_{E}(f[t, B]) \leq \phi\left(t, \alpha_{E}(B)\right)
$$

for any $B \subseteq \bar{B}_{R}\left(x_{0}\right)$ and $t \in[0, T]$, where $\phi$ is a continuous function on $[0, T] \times \mathbf{R}$ with the property that the scalar problem

$$
z^{\prime}(t)=\phi(t, z(t)), \quad z(0)=0,
$$

has only the trivial solution $z(t) \equiv 0$ on $[0, T]$. Then there exists $\tau=\tau\left(x_{0}\right)$ $>0$ such that $(4.7)$ is solvable on $[0, \tau]$.

In recent years, this theorem has been weakened in different ways, often by constructing special new measures of noncompactness (for a sample result see [6]). It is our purpose here to deduce (global) solvability by means of Theorem 3.2. Let $Z=C([0, T] ; E)$ be the space of continuous abstract functions, with norm $\|x\|=\max _{0 \leq t \leq T}\|x(t)\|_{E}$. In this space, the Arzelà-Ascoli criterion reads as follows: A subset $S \subseteq Z$ is relatively compact if and only if it is equicontinuous and the sets $S(t)=\{x(t): x$ $\in S\}$ are relatively compact for all $t \in[0, T]$. Consequently (see [12]), by

$$
\alpha(S)=\max _{0 \leq t \leq T} \alpha_{E}(S(t))
$$

a measure of noncompactness is given on the system of all equicontinuous subsets $S \subset Z$. Now define $X$ to be the subset of all $x \in Z$ such that $x(0)=x_{0}$ and $\left\|x(t)-x_{0}\right\|_{E} \leq R$ for $t \in[0, T]$; then $X$ is a bounded metric space with metric induced by the norm on $Z$. The initial value 
problem (4.7) is equivalent to the fixed point problem $K x=x$, where

$$
K x(t)=x_{0}+\int_{0}^{t} f(s, x(s)) d s \quad(t \in[0, T]),
$$

and, because of (4.8), $K$ maps $X$ into itself.

Let $h=T / n(n \in N)$, and $t_{\nu}=\nu h(\nu=0,1, \ldots, n)$. As in [4], define $K_{n}$ on $X$ by

$$
\begin{aligned}
K_{n} x(t)=x_{0}+h \sum_{\nu=0}^{k-1} f\left(t_{\nu}, x\left(t_{\nu}\right)\right) & +\left(t-t_{k}\right) f\left(t_{k}, x\left(t_{k}\right)\right), \\
t_{k} & \leq t \leq t_{k+1}, k=0,1, \ldots, n-1 .
\end{aligned}
$$

Clearly $K_{n} x(0)=x_{0}$ and $K_{n}(X) \subseteq X$ since

$$
\begin{aligned}
\left\|K_{n} x(t)-x_{0}\right\|_{E} \leq(k+1) h M \leq n h M= & T M \leq R \\
& (x \in X, t \in[0, T]) .
\end{aligned}
$$

Moreover, the continuity of $f$ implies that $K_{n} \rightarrow K$; the uniform continuity of $f$ together with the inequality

$$
\left\|K_{n} x-K_{n} y\right\| \leq T\|F x-F y\|
$$

( $F$ as in (4.3)) imply that $\left[K_{n}\right]$ is equicontinuous. Therefore, as previously observed, $K_{n} \stackrel{c}{\rightarrow} K$. Furthermore, $\bigcup_{n} K_{n}(X)$ is equibounded and, being

$$
\left\|K_{n} x(s)-K_{n} x(t)\right\|_{E} \leq 4 M|s-t|
$$

also equicontinuous. Now assume there exists a continuous function $\gamma$ on $[0, T]$ such that

$$
\alpha_{E}(f[t, B]) \leq \gamma(t) \alpha_{E}(B)
$$

for any $B \subseteq \bar{B}_{R}\left(x_{0}\right)$ and $t \in[0, T]$ (compare this condition with (4.9)). We will show that $K_{n} \stackrel{\lambda-c c}{\rightarrow} K$ with $\lambda=T\|\gamma\|$. Equivalently, we will prove that

$$
\alpha\left(\left[K_{n}\right]\right) \leq \lambda
$$

(the relation $\omega\left(\left[K_{n}\right]\right)=0$ follows again from the fact that $K_{n} \stackrel{c}{\rightarrow} K$ ). To this end, let $S$ be any equicontinuous subset of $X$, and take $\eta>\alpha(S)$. Then, for any $t \in[0, T]$,

$$
\alpha_{E}(F(S(t)))=\alpha_{E}(f[t, S(t)]) \leq \gamma(t) \alpha_{E}(S(t)) \leq \gamma(t) \eta .
$$

Let $\left\{z_{1}(t), \ldots, z_{m}(t)\right\}$ be a $\gamma(t) \eta$-net for $F(S(t))$ in $E$, i.e. for any $x(t) \in S(t)$ there exists $z_{j}(t)$ such that $\left\|f(t, x(t))-z_{J}(t)\right\|_{E} \leq \gamma(t) \eta$. 
Define

$$
Z_{j}(t)=\int_{0}^{t} z_{j}(s) d s+x_{0}
$$

and observe that $\left\{Z_{1}, \ldots, Z_{m}\right\}$ is a $\lambda \eta$-net $(\lambda=T\|\gamma\|)$ for $K(S)$ in $X$, since

$$
\left\|K x(t)-Z_{j}(t)\right\|_{E} \leq \int_{0}^{t}\left\|f(s, x(s))-z_{j}(s)\right\| d s \leq \eta T\|\gamma\| .
$$

This means $\alpha(K(S)) \leq \lambda \alpha(S)$. Moreover, from (4.11) we also deduce $\alpha_{E}\left(K_{n}(S(t))\right) \leq T \gamma(t) \eta$ for all $t \in[0, T]$ and $n \in N$. On the other hand, since $K_{n} \stackrel{c}{\rightarrow} K$, for any $\varepsilon>0$ there exists $n_{\varepsilon} \in N$ such that $K_{n}(S) \subseteq$ $\Omega_{\varepsilon}(K(S))$ for $n \geq n_{\varepsilon}$. Thus

$$
\begin{aligned}
\alpha\left(\bigcup_{n} K_{n}(S)\right) & \leq \max \left\{\alpha\left(K_{1}(S)\right), \ldots, \alpha\left(K_{n_{\varepsilon}}(S)\right), \alpha(K(S))+\varepsilon\right\} \\
& \leq \lambda \alpha(S)+\varepsilon
\end{aligned}
$$

for any $\varepsilon>0$. This shows that $\alpha\left(\left[K_{n}\right]\right) \leq \lambda$, hence $K_{n} \stackrel{\lambda \text {-cc }}{\rightarrow} K$.

Observe now that the same argument as used in [4, p. 606] shows that, for each $n \in N$, the fixed point problem $K_{n} x=x$ has a unique solution $x_{n} \in X$. Therefore, from Theorem 3.2 (iii) (recall that $K_{n} \stackrel{\lambda \text {-cc }}{\rightarrow} K$ implies $K_{n} \stackrel{\lambda \text {-ac }}{\rightarrow} K$ ) we get that $K$ has a fixed point $x \in\left[x_{n}\right]^{*}$ such that, for any $\varepsilon>0$,

$$
\operatorname{dist}\left(x_{n}, \operatorname{Fix}(K)\right) \leq \lambda \alpha\left(\left\{x_{n}\right\}\right)+\varepsilon \quad\left(n \geq n_{\varepsilon}\right) .
$$

Let us give a more suggestive interpretation of this result by distinguishing various cases for the noncompactness of the sequence $\left[K_{n}\right]$ :

(a) $\lambda=0$. This corresponds to the ordinary ac convergence of $\left[K_{n}\right]$; hence, from [4, Theorem 3.7] we get existence of a fixed point, and convergence $x_{n} \rightarrow x$ if $\left[x_{n}\right]^{*}=\{x\}$.

(b) $0<\lambda<1$. In this case we have $\alpha(K)<1$ according to Proposition 3.3 (i), and existence of fixed points follows from Sadovskii's theorem (see $[11,13])$. Moreover, if $\left[x_{n}\right]^{*}=\{x\}$, then the difference $\left\|x_{n}-x\right\|$ is controlled by $\alpha\left(\left\{x_{n}\right\}\right)+\varepsilon\left(n \geq n_{\varepsilon}\right)$; but since $\left[x_{n}\right]^{*}=\{x\}$ implies compactness of $\left\{x_{n}\right\}$, we again have convergence $x_{n} \rightarrow x$.

(c) $\lambda \geq 1$. Now existence of fixed points does not follow any more from fixed point principles for condensing operators, but our Theorem 3.2 (iii) gives $\operatorname{Fix}(K) \neq \varnothing$. On the other hand, the distance of $x_{n}$ to $\operatorname{Fix}(K)$ may now be very large: In fact, it will increase linearly with respect to the noncompactness of both $\left[K_{n}\right]$ and $\left[x_{n}\right]$. 
5. Concluding remarks. Apart from fixed point problems, inhomogeneous problems are also treated in [4]; in this connection, the concept of regular operators and regular convergence plays an important role. In this final section, we shall briefly indicate a possible generalization.

First, let us recall that an operator $A$ between two Banach spaces $X$ and $Y$ is called regular if for any bounded $S \subset X$, the compactness of $\overline{A(S)}$ implies the compactness of $\bar{S}$; a sequence $\left[A_{n}\right]_{N}$ of operators is called asymptotically regular if for any uniformly bounded sequence $\left[S_{n}\right]_{N}$ and all subsets $N^{\prime} \subseteq N$, the $d$-compactness of $\left[A_{n}\left(S_{n}\right)\right]_{N^{\prime}}$ implies the $d$-compactness of $\left[S_{n}\right]_{N^{\prime}}$. A possible generalization of this is to introduce the numbers

$$
\rho(A)=\inf \{\lambda: \lambda>0, \alpha(S) \leq \lambda \alpha(A(S)), S \subset X \text { bounded }\}
$$

and

$$
\begin{array}{r}
\rho\left(\left[A_{n}\right]_{N}\right)=\operatorname{supinf}\left\{\lambda: \lambda>0, \delta\left(\left[S_{n}\right]_{N^{\prime}}\right) \leq \lambda \delta\left(\left[A_{n}\left(S_{n}\right)\right]_{N^{\prime}}\right),\right. \\
\left.\left[S_{n}\right]_{N} \text { uniformly bounded }\right\},
\end{array}
$$

where the supremum is taken over all infinite subsets $N^{\prime} \subseteq N$. Further, we introduce a type of convergence which generalizes the regular convergence in [4]; given $\lambda \geq 0$,

$$
\begin{aligned}
A_{n} \stackrel{\lambda-\mathrm{r}}{\rightarrow} A & (\lambda \text {-regular convergence }) \text { means } \\
& A_{n} \stackrel{c}{\rightarrow} A \text { and } \rho\left(\left[A_{n}\right]_{N}\right) \leq \lambda .
\end{aligned}
$$

As a motivation for this generalization, we give a result on $\lambda$-r convergent operator sequences which contains in part Theorem 4.10 in [4] and plays the same role for inhomogeneous equations $A x=y$ as Theorem 3.3 for fixed point equations $K x=x$.

THeOrem 5.1. Let $A_{n}, A$ be operators between $X$ and $Y$ and let $A_{n} \stackrel{\lambda-\mathrm{r}}{\rightarrow} A$. Let $\left[S_{n}\right]_{N}$ be a sequence of subsets in $X$ such that $\cup_{n} S_{n}$ is bounded, and let $\left[y_{n}\right]_{N}$ be a bounded sequence in $Y$. Define

$$
\tilde{S}_{n}=\left\{x: x \in S_{n}, A_{n} x=y_{n}\right\} .
$$

Then

(i) $\delta\left(\left[\tilde{S}_{n}\right]_{N}\right) \leq \delta\left(\left[y_{n}\right]_{N}\right)$;

(ii) $\left[y_{n}\right]_{N}^{*} \neq \varnothing$ implies $\left[\tilde{S}_{n}\right]_{N}^{*} \neq \varnothing$ and $A\left(\left[\tilde{S}_{n}\right]_{N}^{*}\right) \subseteq\left[y_{n}\right]_{N}^{*}$. 
Proof. (i) From $A_{n} \stackrel{\lambda-r}{\rightarrow} A$ it follows that

$$
\delta\left(\left[\tilde{S}_{n}\right]\right) \leq \lambda \delta\left(\left[A_{n}\left(\tilde{S}_{n}\right)\right]\right)=\lambda \delta\left(\left[y_{n}\right]\right)
$$

by definition of $\tilde{S}_{n}$.

(ii) If $\left[y_{n}\right]^{*} \neq \varnothing$, then $\delta\left(\left[y_{n}\right]\right)<\infty$, hence $\delta\left(\left[\tilde{S}_{n}\right]\right)<\infty$, hence $\left[\tilde{S}_{n}\right]^{*}$ $\neq \varnothing$. Moreover, given $x \in\left[\tilde{S}_{n}\right]^{*}$ and $x_{n} \in \tilde{S}_{n}$ such that $x_{n} \rightarrow x\left(n \in N^{\prime}\right)$, we have $y_{n}=A_{n} x_{n} \rightarrow A x\left(n \in N^{\prime}\right)$ which means $A x \in\left[y_{n}\right]^{*}$.

Regular convergence has applications to boundary value problems for nonlinear differential equations (see [4, Example 4.2]). However, there seems to be another field of possible applications, namely nonlinear spectral theory. The reader can find some interesting topics on nonlinear spectral theory, for example, in [7]; in this paper the number

$$
\beta(A)=\sup \{\lambda: \lambda>0, \alpha(A(S)) \geq \lambda \alpha(S), S \subset X \text { bounded }\}
$$

plays a crucial role for the definition and description of the spectrum of a nonlinear operator $A$. It is not hard to see that $\beta(A)=1 / \rho(A)$; consequently, all results involving the number $\beta(A)$ can be expressed equivalently in terms of $\rho(A)$. (To give a simple example, a continuous linear operator $A$ between two Banach spaces $X$ and $Y$ is Fredholm if and only if $\rho(A)$ and $\rho\left(A^{*}\right)$ are both finite, where $A^{*}$ is the adjoint of $A$.)

Some connections between nonlinear spectral theory and regular operator convergence will be discussed in a forthcoming paper.

Note added in proof. After submitting the present paper, the authors became acquainted with G. M. Vainikko's work on measures of discrete noncompactness (see [18-21] and, in particular, [22]). Our measure $\delta$, however, is defined in another way, and has different properties. We thank Professor Vainikko for pointing our attention to the papers [18-22].

\section{REFERENCES}

[1] R. R. Ahmerov, M. I. Kamenskii, A. S. Potapov, and B. N. Sadovskii, Condensing operators, (Russian), Itogi Nauki Tech., 18 (1980), 185-250.

[2] R. R. Ahmerov, M. I. Kamenskii, A. S. Potapov, A. E. Rodkina, and B. N. Sadovskii, Theory of equations of neutral type, (Russian), Itogi Nauki Tech., 19 (1982), 55-126.

[3] P. M. Anselone, Collectively Compact Operator Approximation Theory, Prentice-Hall, Englewood Cliffs (1971).

[4] P. M. Anselone and R. Ansorge, Compactness principles in nonlinear operator approximation theory, Numer. Funct. Anal. Optim., 1 (6) (1979), 589-618.

[5] J. Appell, Implicit functions, nonlinear integral equations, and the measure of noncompactness of the superposition operator, J. Math. Anal. Appl., 83, 1 (1981), 251-263. 
[6] J. Banás, A. Hajnosz, and S. Wedrychowicz, On the equation $x^{\prime}=f(t, x)$ in Banach spaces, Comm. Math. Univ. Carol., 23, 2 (1982), 233-247.

[7] M. Furi, M. Martelli, and A. Vignoli, Contributions to the spectral theory for nonlinear operators in Banach spaces, Ann. Mat. Pura Appl., (IV) 118 (1978), 229-294.

[8] Z. Grande and J. S. Lipiński, Un exemple d'une fonction sup-mesurable qui n'est pas mesurable, Coll. Math., 39, 1 (1978), 77-79.

[9] Z. Grande, La mesurabilité des fonctions de deux variables et de la superposition $F(x, f(x))$, Diss. Math., 159 (1979).

[10] A. Istrătescu and V. Istrătescu, A generalization of collectively compact sets of operators, Rev. Roumaine Math. Pures Appl., 17 (1972), 33-37.

[11] B. N. Sadovskii, On measures of noncompactness and condensing operators, (Russian), Probl. Mat. Anal. Slozh. Sistem Voronezh, 2 (1968), 89-119.

[12] On the local solvability of ordinary differential equations in Banach spaces, (Russian), Probl. Mat. Anal. Slozh. Sistem Voronezh, 3 (1968), 232-243.

[13] Limit-compact and condensing operators, (Russian), Usp. Mat. Nauk, 27 (1972), 81-146.

[14] I. V. Shragin, Conditions for the measurability of the superposition, (Russian), Dokl. Akad. Nauk SSSR, 197 (1971), 295-298.

[15] __ Conditions for the convergence of superpositions, (Russian), Diff. Uravn., 13 (1977), 1900-1901.

[16] R. Wolf, Diskret kondensierende Operatorfolgen, Math. Nachr., 80 (1977), 209-233.

[17] _ Approximation of fixed points of condensing mappings, Appl. Anal., 9 (1979), $125-136$.

[18] G. M. Vainikko, Analysis of discretization methods, (Russian), Tartu, 1976.

[19] __ Funktionalanalysis der Diskretisierungsmethoden, Leipzig, 1976.

[20] —ü Über Konvergenzbegriffe für lineare Operatoren in der Numerischen Mathematik, Math. Nachr., 78 (1977), 165-183.

[21] __ Approximative methods for nonlinear equations (two approaches to the convergence problem). Nonlin. Anal., 2, 6, (1978), 647-687.

[22] Discrete measures of noncompactness (Russian), Matemaatilise Füüsika Ülesannete Lahendusmeetodid, Tartu riikliku ülikooli toimetised, vihik, 580, (1981), $3-8$.

Received January 28, 1983. Work of the first author was performed under the auspices of the Italian National Research Council (CNR) while the first author was visiting professor at the University of Florence.

UNIVERSITA DI FIRENZE

50134 FiRENZE, ITALY 



\title{
PACIFIC JOURNAL OF MATHEMATICS \\ EDITORS
}

\author{
DoNALD BABBITT (Managing Editor) \\ University of California \\ Los Angeles, CA 90024 \\ J. DugunduI \\ University of Southern California \\ Los Angeles, CA 90089-1113 \\ R. FINN \\ Stanford University \\ Stanford, CA 94305 \\ HERMANN FlasChKa \\ University of Arizona \\ Tucson, AZ 85721
}

C. C. Moore

University of California

Berkeley, CA 94720

ARTHUR OGUS

University of California

Berkeley, CA 94720

Hugo Rossi

University of Utah

Salt Lake City, UT 84112

H. SAMELSON

Stanford University

Stanford, CA 94305

ASSOCIATE EDITORS
R. ARENS
E. F. BECKENBACH
B. H. NeUMANN
F. WOLF
K. YoSHIDA (1906-1982)

\section{SUPPORTING INSTITUTIONS}

UNIVERSITY OF ARIZONA
UNIVERSITY OF BRITISH COLUMBIA
CALIFORNIA INSTITUTE OF TECHNOLOGY
UNIVERSITY OF CALIFORNIA
MONTANA STATE UNIVERSITY
UNIVERSITY OF NEVADA, RENO
NEW MEXICO STATE UNIVERSITY
OREGON STATE UNIVERSITY
UNIVERSITY OF ARIZONA
CALIFORNIA INSTITUTE OF TECHNOLOGY
UNIVERSITY OF CALIFORNIA
UNIVERSITY OF NEVADA, RENO
OREGON STATE UNIVERSITY
UNIVERSITY OF OREGON
UNIVERSITY OF SOUTHERN CALIFORNIA
STANFORD UNIVERSITY
UNIVERSITY OF HAWAII
UNIVERSITY OF TOKYO
UNIVERSITY OF UTAH
WASHINGTON STATE UNIVERSITY
UNIVERSITY OF WASHINGTON 


\section{Pacific Journal of Mathematics}

Vol. 115, No. $1 \quad$ September, 1984

Carlos Andradas Heranz and José Manuel Gamboa Mutuberría, A note on projections of real algebraic varieties $\ldots \ldots \ldots \ldots \ldots \ldots \ldots \ldots \ldots \ldots$

Jürgen Appell and Maria Patrizia Pera, Noncompactness principles in nonlinear operator approximation theory $\ldots \ldots \ldots \ldots \ldots \ldots \ldots \ldots \ldots \ldots$

Timothy John Carlson, Extending Lebesgue measure by infinitely many

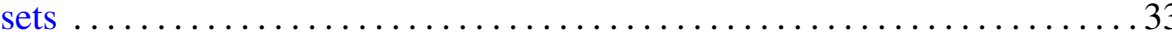

Donald S. Coram and Paul Frazier Duvall, Jr., Non-cell-like

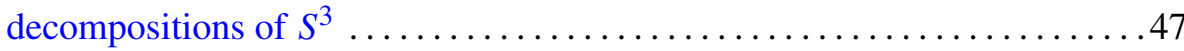

Edward Norman Dancer, Order intervals of selfadjoint linear operators and

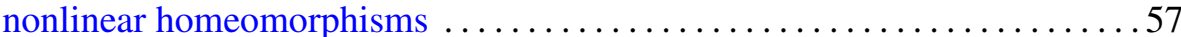

Ralph Jay De Laubenfels, Well-behaved derivations on $C[0,1] \ldots \ldots \ldots 73$

D. Feyel and A. de La Pradelle, Sur certaines extensions du théorème

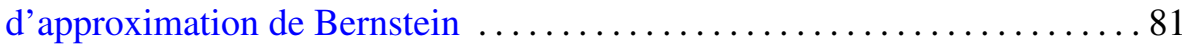

Colin C. Graham and Bertram Manuel Schreiber, Bimeasure algebras on

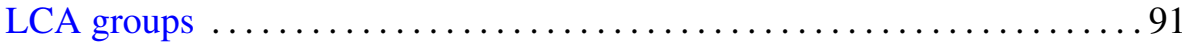

Richard Howard Hudson, Class numbers of imaginary cyclic quartic fields

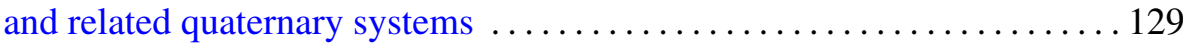

Carl Groos Jockusch, Jr. and Iraj Kalantari, Recursively enumerable sets and van der Waerden's theorem on arithmetic progressions . . . . . . . . 143

J. F. McClendon, On noncontractible valued multifunctions ........... 155 Akihiko Miyachi, Weak factorization of distributions in $H^{p}$ spaces $\ldots \ldots \ldots 165$ Ezzat S. Noussair and Charles Andrew Swanson, Global positive solutions of semilinear elliptic problems

Jon Christopher Snader, Strongly analytic subspaces and strongly

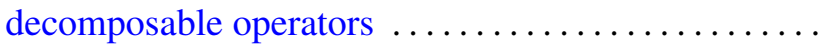

Boguslaw Tomaszewski, A construction of inner maps preserving the Haar measure on spheres

Akihito Uchiyama, The Fefferman-Stein decomposition of smooth functions and its application to $H^{p}\left(\mathbf{R}^{n}\right) \ldots \ldots \ldots$ 